\title{
A Result Related To The Value Distribution Of Gamma Functions.
}

\author{
K. S. L. N. Prasad \\ Associate professor,Dept.of Mathematics, Karnatak Arts College, Dharwad-580001.
}

Abstract: In this paper we have extended a result of Nevanlinna theory to Euler's gamma function which is known to be a meromorphic function.

Key Words: Nevanlinna theory, Euler's gamma function.

\section{Introduction And Main Results}

Let $\Gamma(\mathrm{z})$ be the Euler's gamma function defined by,

$$
\Gamma(\mathrm{z})=\frac{\mathrm{e}^{-v \mathrm{z}}}{\mathrm{z}} \prod_{\mathrm{k}=1}^{\infty}\left(1+\frac{\mathrm{z}}{\mathrm{k}}\right)^{-1} \mathrm{e}^{\frac{\mathrm{z}}{\mathrm{k}}}
$$

Where $\gamma=\lim _{\mathrm{n} \rightarrow \infty} \sum_{\mathrm{k}=1}^{\mathrm{n}}\left(\frac{1}{\mathrm{k}}-\log \mathrm{n}\right)$, the Euler's constant

Clearly $\Gamma(\mathrm{z})$ is a meromorphic function with simple poles $\{-\mathrm{k}\}_{\mathrm{k}=0}^{\infty}$ and $\Gamma(\mathrm{z}) \neq 0$ for any $\mathrm{z} \in \mathrm{C}$.

In 1999, Zhuan Ye[2] has proved the following result.

Theorem (A) : with usual notations,
i) $\mathrm{T}(\mathrm{r}, \Gamma)=(1+\mathrm{o}(1)) \frac{\mathrm{r}}{\pi} \log \mathrm{r}$
ii) $\delta(\mathrm{o}, \Gamma)=1, \quad \delta(\infty, \Gamma)=1$
iii) $\delta(\mathrm{a}, \Gamma)=0$ for $\mathrm{a} \neq 0, \infty$

Proceeding on the same lines, we can observe the following.

$$
\text { since } \delta(\mathrm{a}, \Gamma)=0 \quad \text { for } \mathrm{a} \neq 0, \infty \text { and } \delta(\mathrm{a}, \Gamma)=1 \text { and } \delta(\infty, \Gamma)=1 \text {, }
$$

Using the basics of Nevalinna theory, we can easily prove that,

i) $\quad \Theta(a, \Gamma)=0 \quad$ for $a \neq 0, \infty$

ii) $\quad \Theta(0, \Gamma)=0$ and $\Theta(\infty, \Gamma)=1$.

We wish to establish the following result.

Theorem : Let $\Gamma(\mathrm{z})$ be the Euler's gamma function.Then

$\mathrm{T}\left(\mathrm{r}, \Gamma^{(\mathrm{n})}\right) \sim(\mathrm{n}+1) \mathrm{T}(\mathrm{r}, \Gamma)$ where $\mathrm{n}$ is any positive integer, as $\mathrm{r} \rightarrow \infty$ outside a set of finite linear measure.

Proof: Clearly $\Gamma(\mathrm{z})$ is a meromorphic function with simple poles $\{-\mathrm{k}\}_{\mathrm{k}=0}^{\infty}$ and $\Gamma(\mathrm{z}) \neq 0$ for any $\mathrm{z} \in \mathrm{C}$.

Therefore,

$$
\overline{\mathrm{N}}\left(\mathrm{r}, \frac{1}{\Gamma}\right)=0
$$

Using the basics of Nevanlinna theory, we have $\mathrm{m}\left(\mathrm{r}, \frac{\Gamma^{\dagger}}{\Gamma}\right) \leq 0\{\log \mathrm{T}(\mathrm{r}, \Gamma)+\mathrm{O}(\log \mathrm{r})$ as $\mathrm{r} \rightarrow \infty$ outside a set of finite linear measure.

By induction on $\mathrm{n}$, we can prove that, 
$\mathrm{m}\left(\mathrm{r}, \frac{\Gamma^{(\mathrm{n}) \mid}}{\Gamma}\right) \leq 0\{\log \mathrm{T}(\mathrm{r}, \Gamma)+\mathrm{O}(\log \mathrm{r})$ for all finite $\mathrm{n}$.

Since $\mathrm{N}\left(\mathrm{r}, \frac{\Gamma^{(\mathrm{n}) \mid}}{\Gamma}\right) \quad=\mathrm{n} \overline{\mathrm{N}}(\mathrm{r}, \Gamma)+\overline{\mathrm{N}}\left(\mathrm{r}, \frac{1}{\Gamma}\right)$

$$
=\mathrm{n} \overline{\mathrm{N}}(\mathrm{r}, \Gamma), \quad \text { since } \overline{\mathrm{N}}\left(\mathrm{r}, \frac{1}{\Gamma}\right)=0 \text {. }
$$

We have, $\left.\mathrm{T}\left(\mathrm{r}, \frac{\Gamma^{(\mathrm{n})}}{\Gamma}\right) \leq \mathrm{n} \overline{\mathrm{N}}(\mathrm{r}, \Gamma)+\mathrm{O}\{\log \mathrm{T}(\mathrm{r}, \Gamma)\}+\mathrm{O}(\log \mathrm{r})\right\}$

Then, $\quad \mathrm{T}\left(\mathrm{r}, \Gamma^{(\mathrm{n})}\right) \quad=\mathrm{T}\left(\mathrm{r}, \frac{\Gamma^{(\mathrm{n})}}{\Gamma}, \Gamma\right)$

$$
\begin{gathered}
\leq \mathrm{T}\left(\mathrm{r}, \frac{\Gamma^{(\mathrm{n})}}{\Gamma}\right)+\mathrm{T}(\mathrm{r}, \Gamma) \\
\leq \mathrm{T}(\mathrm{r}, \Gamma)+\mathrm{n} \overline{\mathrm{N}}(\mathrm{r}, \Gamma)+\mathrm{O}\{\{\log \mathrm{T}(\mathrm{r}, \Gamma)\}+\mathrm{O}(\log \mathrm{r})\}
\end{gathered}
$$

Conversely, $\quad \mathrm{T}(\mathrm{r}, \Gamma)=\mathrm{T}\left(\mathrm{r}, \frac{\Gamma^{(\mathrm{n})}}{\Gamma}, \Gamma^{(\mathrm{n})}\right)$

$$
\begin{aligned}
& \leq \mathrm{T}\left(\mathrm{r}, \frac{\Gamma}{\Gamma^{(\mathrm{n})}}\right)+\mathrm{T}\left(\mathrm{r}, \Gamma^{(\mathrm{n})}\right)+\mathrm{O}(1) \\
& \quad \leq \mathrm{T}\left(\mathrm{r}, \Gamma^{(\mathrm{n})}\right)+\mathrm{n} \overline{\mathrm{N}}(\mathrm{r}, \Gamma)+\mathrm{O}\{\log \mathrm{T}(\mathrm{r}, \Gamma)\}+\mathrm{O}(\log \mathrm{r})
\end{aligned}
$$

From (1) and (2), We have

$\left|\mathrm{T}\left(\mathrm{r}, \Gamma^{(\mathrm{n})}\right)-\mathrm{T}(\mathrm{r}, \Gamma)\right| \leq \mathrm{n} \overline{\mathrm{N}}(\mathrm{r}, \Gamma)+\mathrm{O}\{\log \mathrm{T}(\mathrm{r}, \Gamma)\}+\mathrm{O}(\log \mathrm{r})$

On simplification, we get $\lim _{\mathrm{n} \rightarrow \infty} \frac{\mathrm{T}\left(\mathrm{r}, \Gamma^{(\mathrm{n})}\right)}{\mathrm{T}(\mathrm{r}, \Gamma)}=\mathrm{n}+1$

Hence the result.

$$
\text { Or } \quad \mathbf{T}\left(\mathbf{r}, \Gamma^{(\mathrm{n})}\right) \sim(\mathbf{n}+\mathbf{1}) \mathbf{T}(\mathbf{r}, \Gamma)
$$

\section{References}

[1] HAYMAN W. K. (1964) : Meromorphic functions, Oxford Univ. Press, London.

[2] ZHUAN YE (1999) : Note - The Nevanlinna functions of the Riemann Zeta function, Jl. of math. ana. and appl. 233, 425-435. 